\title{
Intense Pulsed Light Combined With Meibomian Gland Massage for Recurrent Corneal Erosion: a Review of Clinical Effectiveness
}

\author{
Man Xu \\ Aier eye hospital of Wuhan university \\ Xiaoguang Niu ( $\sim 13476186008 @ 163 . c o m$ ) \\ Aier eye hospital of Wuhan university
}

\section{Research Article}

Keywords: recurrent corneal erosion syndrome (RCES), Intense pulsed light, Meibomian gland massage, clinical effectiveness

Posted Date: January 12th, 2022

DOI: https://doi.org/10.21203/rs.3.rs-1219232/v1

License: () (7) This work is licensed under a Creative Commons Attribution 4.0 International License. Read Full License 


\section{Abstract}

Purpose: To observe the therapeutic effect on recurrent corneal erosion syndrome (RCES) by intense pulsed light (IPL) combined with meibomian gland massage.

Methods: We recruited 30 patients (30 eyes) with RCES as the RCES group and 31 patients (31 eyes) as the control group. Both the groups received eyelash sampling, Demodex count, meibomian gland infrared photography, and the blepharolipin score. The RCES group was divided into the treatment group and the observation group again.

Results: Parameters in the RCES group were higher than those in the control group. The parameters of the treatment group were decreased compared with the observation group. During the follow-up period, one patient in the treatment group relapsed.

Conclusion: Meibomian gland dysfunction and Demodex infection may be associated with RCES. IPL combined with meibomian gland massage can significantly improve meibomian gland function, reduce the number of Demodex, and effectively control the relapse of RCES.

\section{Background}

Recurrent corneal erosion syndrome (RCES) appears to be one of the common diseases of the ophthalmic clinic, acknowledged as corneal epithelial basement membrane and corneal stroma cannot be attached firmly ${ }^{[1]}$ caused by kinds of reasons. The corneal epithelium repeatedly exfoliated the relapse of the disease that affected the patients' work and life, bringing them severe physical and psychological troubles. The etiology of RCES includes corneal epithelial basement membrane dystrophy, corneal trauma, herpetic keratopathy, meibomian gland dysfunction etc ${ }^{[2,3]}$. Meibomian gland dysfunction (MGD) refers to a chronic or diffuse lesion of the meibomian gland characterized by terminal gland duct obstruction, abnormal secretion of the gland and/or quantity. It makes the tear film unstable, ocular surface irritation symptoms, with or without inflammation, along with other ocular surface diseases. MGD causes a variety of ocular surface diseases. It indicates that MGD may be the leading cause of RCES above all and there is a vital correlation between MGD and RCES ${ }^{[4]}$. The infection rate and the number of Demodex on eyelash are high in MGD patients; Demodex infection exactly performs huge effect on MGD ${ }^{[5]}$. However, whether Demodex infection involved in the pathogenesis of RCES remains unreported. While whether the treatment for MGD can control RCES has not been reported either.

Our study conducts the detection of Demodex infection and meibomian gland in RCES patients to observe whether Demodex infection and the degree of the meibomian gland dysfunction are related to RCES. Simultaneously intensive pulsed light (IPL) combined with meibomian gland massage is applied to treat these patients, observe whether their symptoms and recurrence frequency are controlled after the treatment.

\section{Methods}

\section{Studying Objects}

This is a prospective case-control study. We screened 30 patients (30 eyes) with two or more recurrent RCEs in our hospital from June 2018 to December 2018 , including 17 males and 13 females, with an average age of 44.45 \pm .75(32 58)years old. Besides, thirty-one patients (31 eyes) with matched gender, age, with no other ocular surface diseases were selected from the optometry clinic as the normal-control group, including 20 males and 11 females, with an average age of $44.81 \pm 9.40$ (33 60) years old. There was no significant difference statistically between the two groups in gender distribution and age $(t=0.62, P=0.54$, respectively; $\mathrm{t}=0.18, \mathrm{P}=0.86)$. The exclusion criteria includes: corneal infection, corneal scar can be seen with slit-lamp, bilateral ocular disease, intraocular hypertension, dacryocystitis, intraocular inflammation, along with diabetes mellitus and systemic immune diseases. Both patients of the two groups received anterior ocular photography, Demodex exam by eyelash microscopic examination, and taken infrared photography of meibomian gland infrared with an ocular surface comprehensive analyzer. This study was approved by the ethics committee of Aier eye hospital of Wuhan University (approval no. HYEYE2018IRB01). The same physician conducted all the examinations, and the informed consent form has been signed, but the Patients or the public were not involved in the design, or conduct, or reporting, or dissemination plans of our research.

\section{Examinations and Record Methods}

1. Gender, age, time of trauma, and exacerbation frequency of RECS patients were recorded in detail.

2. The score of the loss of the meibomian [6]: the examination was conducted by the same senior technician with Keratograph $5 \mathrm{M}$ ocular synthesis analyzer (German Oculus, TYP 77000), where the brightness of indoor light source was keeping consistent. During the examination, put the patient's lower jaw up in the bracket, eyes parallel to the black ring of the bracket, lifted the upper and lower lids separately, made the conjunctiva fully exposed, and take the photograph. Evaluate the meibomian scores: 0 point for normal (no loss of the meibomian gland); 1 point for the loss of the meibomian gland was less than 1/3; 2 point for the loss of the meibomian gland was between 1/3 2/3. Three points for the loss of the meibomian gland was greater than $2 / 3$. Based on both the upper and lower meibomian glands' overall scores, each eye's score is 0 to 6 points.

3. Blepharolipin score ${ }^{[6]}$ : let the patient look up, press the 8 Meibomian glands on the middle $1 / 3$ area of the lower eyelid with moderate pressure, evaluate the secretion characteristics of the Meibomian gland, 0-24 point in total. 0 for crystal clear normal liquid meibum; 1 for muddy meibum; 2 for granular liquid meibum; 3 for toothpaste-like thick meibum.

4. Lid margin abnormality score ${ }^{[6]}$ : The eyelid margin morphology was observed under a slit lamp. There were four physical signs: Irregularity of eyelid margin, eyelid marginal hyperemia, Meibomian gland block, The mucosal skin boundary is displaced. None of the four signs, the score was zero, 1 point for 1 sign; the total score was 0-4 point. 
5. Demodex examination of eyelashes ${ }^{[7]}$ : pluck 3 thick eyelashes from the middle $1 / 3$ of the upper and lower eyelid each eye (6 eyelashes in all), put them on the microslide, covered with a coverslip, add saline from one side, observe the Demodex under the photoelectric microscope, and count the number.

\section{Therapeutic Method}

All the patients were treated with antibiotic spongarion and artificial tears eye drop after the first-time consultancy. After two weeks of eye drop treatment, they were randomly divided into two groups, the patients in the treatment group treated with IPL combined with meibomian gland massage three times, once every 28 days; The patients in the observation group treated with preservative-free artificial tears eye drop only.

We used SOLARI (Lutronic, Korea) intense pulse optical equipment for the treatment; parameters were tested in the patient's jaw before the first treatment, adjust the IPL energy according to the patient's skin color, the energy range was $9813 \mathrm{~J} / \mathrm{cm}^{2}$, kept the patient's skin clean and dry, and wore an eye mask to protect the patient's eyes. The treatment area was uniformly coated with a medical coupling agent for $5 \sim 10 \mathrm{~mm}$. Doctor was expected to wear goggles during the whole treatment. Four therapeutic parts were selected from the left temple to the right, putting the probe on the coupling agent part, press the on-off button to emit the pulses of light, then move to the next part, and redo the operation until all the therapeutic parts have been done. When the treatment has been finished, remove the skin coupling gel, drop $0.4 \%$ bupivacaine hydrochloride (Santen Pharmaceutical co. LTD Japan) into the conjunctival sac two times, once every 5 minutes, then massaged the upper and lowered meibomian gland with meibomian gland massage tweezers. The same physician performed all procedures. When all treatment has been finished, ice the eyes for 5 minutes.

\section{Therapeutic Evaluation}

After the accomplishment of three treatments, Compared the meibomian gland loss score, blepharolipin score, the number of Demodex, and the disease recurrence during the one-year-follow-up period in the two groups respectively.

\section{Statistical Analysis}

SPSS22.0 (SPSS, USA) was used for statistical analysis. In this study, all counting data were skewed by the shapiro-wilk test and expressed by M (Q1, Q3). The Mann-Whitney U test was used to compare the meibomian gland loss score, the blepharolipin score, and the number of Demodex in the two groups. The chi-square test was used to compare the positive detection rate of granular Demodex and the recurrence rate of the disease, $\mathrm{P}<0.05$ was considered significant differences statistically.

\section{Results}

1. Compare the loss of Meibomian gland score and the blepharolipin score

There was hyperemia of the eyelid margin and blockage of Meibomian gland openings in varying degrees of all the 30 RCES patients; at the root of their eyelashes, there were scales and cuff-like secretions attached, and Meibomian gland glands were tortuous, shortened, and absent. The Meibomian gland function of RCES patients was significantly lower than that of the control group. $(P<0.01)$ (Table 1$)$

Table 1

comparison of the correlated parameters of demodex and meibomian gland between RCES group and normal control group

\begin{tabular}{|lllll|}
\hline Groups & Numbers & $\begin{array}{l}\text { the meibomian gland loss score } \\
{\left[M\left(Q_{1}, Q_{3}\right)\right]^{*}}\end{array}$ & $\begin{array}{l}\text { blepharolipin score }\left[M\left(Q_{1},\right.\right. \\
\left.\left.Q_{3}\right)\right]^{*}\end{array}$ & $\begin{array}{l}\text { lid margin abnormality } \\
\text { score }\left[M\left(Q_{1}, Q_{3}\right)\right]\end{array}$ \\
\hline RCES & 30 & $4(3.0,4.0)$ & $15.5(11.0,16.8)$ & $3(2.8,3.0)$ \\
\hline Control & 31 & $2(1.0,3.0)$ & $8.0(5.5,10.0)$ & $2(1.0,3.0)$ \\
$Z / X^{2}$ & & $Z=-4.55$ & $Z=-3.97$ & $Z=-4.22$ \\
$P$ & $P<0.01$ & $P<0.01$ & $P<0.01$ & $38.7 \%$ \\
$X^{2}=7.60$ & $P<0.01$ \\
\hline
\end{tabular}

\section{Compare the Demodex infection}

The detection rate of Demodex was $83.3 \%$ in patients with recurrent corneal epithelial erosion and $38.7 \%$ in the control group, with a significant difference between the 2 groups $\left(\chi^{2}=7.60, P=0.00\right)$ (Table 1$)$. In the recurrent corneal erosion group, the maximum number of Demodex mites in one eye was 18 , while in the control group, the maximum number of Demodex mites in one eye were 8 .

\section{Therapeutic Effect}

There were 16 patients (16 eyes) including 9 males and 7 females in the treatment group, and 14 patients (14 eyes) including 8 males and 6 females in the observation group; there was no significant difference in age and gender the two groups. In the treatment group, after 3 times of IPL combined with meibomian gland massage treatments, the posterior eyelid congestion was reduced, the attachment of the secretion from the eyelash root was significantly reduced, the meibomian gland loss score and the blepharolipin score were all lower than before, and the number of Demodex and the detection rate of Demodex were significantly lower than before (Table 2). There was no significant change in the observation group. 
Table 2

The correlated parameters in IPL treatment group before and after treatment

\begin{tabular}{|c|c|c|c|c|c|c|c|c|c|c|c|c|}
\hline & \multirow[b]{2}{*}{ Age } & \multirow[b]{2}{*}{ Sex } & \multirow[b]{2}{*}{ Eye } & \multicolumn{5}{|c|}{ before treatment } & \multicolumn{4}{|c|}{ after treatment } \\
\hline & & & & Recurrent & $\begin{array}{l}\text { Number } \\
\text { of } \\
\text { demodex }\end{array}$ & $\begin{array}{l}\text { Blepharolipin } \\
\text { score }\end{array}$ & $\begin{array}{l}\text { the } \\
\text { meibomian } \\
\text { gland loss } \\
\text { score }\end{array}$ & $\begin{array}{l}\text { lid margin } \\
\text { abnormality } \\
\text { score }\end{array}$ & $\begin{array}{l}\text { Number } \\
\text { of } \\
\text { demodex }\end{array}$ & $\begin{array}{l}\text { Blepharolipin } \\
\text { score }\end{array}$ & $\begin{array}{l}\text { the } \\
\text { meibomian } \\
\text { gland loss } \\
\text { score }\end{array}$ & $\begin{array}{l}\text { lid margin } \\
\text { abnormality } \\
\text { score }\end{array}$ \\
\hline 1 & 58 & M & $L$ & 8 & 18 & 20 & 4 & 4 & 3 & 12 & 4 & 2 \\
\hline 2 & 54 & M & $\mathrm{R}$ & 4 & 4 & 10 & 3 & 3 & 0 & 5 & 3 & 2 \\
\hline 3 & 55 & M & $\mathrm{L}$ & 3 & 9 & 18 & 4 & 3 & 6 & 10 & 4 & 1 \\
\hline 4 & 52 & M & $L$ & 2 & 8 & 16 & 4 & 2 & 5 & 10 & 4 & 2 \\
\hline 5 & 46 & $\mathrm{~F}$ & $L$ & 3 & 5 & 8 & 2 & 3 & 0 & 4 & 1 & 1 \\
\hline 6 & 44 & M & $\mathrm{R}$ & 2 & 8 & 17 & 5 & 3 & 4 & 16 & 4 & 1 \\
\hline 7 & 54 & $\mathrm{~F}$ & $\mathrm{~L}$ & 3 & 8 & 16 & 4 & 4 & 0 & 8 & 3 & 3 \\
\hline 8 & 33 & $\mathrm{M}$ & $L$ & 3 & 10 & 10 & 4 & 4 & 2 & 10 & 4 & 2 \\
\hline 9 & 42 & $M$ & $\mathrm{R}$ & 3 & 0 & 8 & 3 & 2 & 0 & 6 & 2 & 1 \\
\hline 10 & 34 & $M$ & $\mathrm{~L}$ & 2 & 4 & 16 & 4 & 3 & 0 & 12 & 4 & 1 \\
\hline 11 & 29 & $\mathrm{~F}$ & $\mathrm{~L}$ & 5 & 8 & 17 & 4 & 2 & 2 & 12 & 4 & 2 \\
\hline 12 & 31 & $\mathrm{M}$ & $L$ & 2 & 4 & 10 & 3 & 2 & 0 & 8 & 3 & 1 \\
\hline 13 & 62 & $\mathrm{~F}$ & $L$ & 6 & 9 & 18 & 4 & 4 & 3 & 15 & 4 & 3 \\
\hline 14 & 32 & $\mathrm{~F}$ & $\mathrm{R}$ & 3 & 8 & 16 & 4 & 2 & 1 & 10 & 4 & 1 \\
\hline 15 & 35 & $\mathrm{~F}$ & $\mathrm{~L}$ & 5 & 0 & 8 & 2 & 3 & 0 & 8 & 1 & 2 \\
\hline 16 & 36 & $\mathrm{~F}$ & $\mathrm{~L}$ & 2 & 18 & 20 & 5 & 3 & 5 & 12 & 4 & 2 \\
\hline
\end{tabular}

1 patient in the treatment group had relapsed follow-up for one year after treatment, 5 patients had slight foreign body sensation occasionally. In patients with foreign body sensation, the part of the corneal, which the epithelium erosion repeatedly, showed punctuate opaque but not detached. (Figure1) Moreover, in the observation group, 6 patients had relapsed. The difference between the two groups was statistically significant $\left(\chi^{2}=5.04, P=0.04\right)$. (Table 3$)$

Table 3

Comparison of correlated parameters of demodex and meibomian gland before and after treatment in IPL treatment group

\begin{tabular}{|c|c|c|c|c|c|c|}
\hline Groups & Numbers & $\begin{array}{l}\text { the meibomian gland loss } \\
\text { score }\left[M\left(Q_{1}, Q_{3}\right)\right]^{\star}\end{array}$ & $\begin{array}{l}\text { blepharolipin } \\
\text { score }\left[M\left(Q_{1}, Q_{3}\right)\right]^{*}\end{array}$ & $\begin{array}{l}\text { lid margin abnormality } \\
\text { score }\left[M\left(Q_{1}, Q_{3}\right)\right]\end{array}$ & $\begin{array}{l}\text { number of demodex } \\
{\left[M\left(Q_{1}, Q_{3}\right)\right]^{*}}\end{array}$ & $\begin{array}{l}\text { Positive rate of } \\
\text { demodex }\end{array}$ \\
\hline Before & 16 & $4.0(3,4)$ & $16.0(10.0,17.8)$ & $3.0(2.0,3.8)$ & $8.0(4,9)$ & $87.5 \%$ \\
\hline After & 16 & $4.0(3,4)$ & $10.0(8 \cdot 0,12.0)$ & $2.0(1.0,2.0)$ & $3.0(0,4.5)$ & $56.3 \%$ \\
\hline$z / \chi^{2}$ & & $Z=-2.45$ & $Z=-3.31$ & $Z=-3.40$ & $Z=-5.01$ & $x^{2}=3.87$ \\
\hline$P$ & & $P=0.01$ & $P=0.00$ & $P=0.01$ & $P=0.00$ & $P=0.11$ \\
\hline
\end{tabular}

\section{Discussion}

In 1994, Hope-Ross MW ${ }^{[4]}$ has researched 30 patients with refractory recurrent corneal epithelial erosion with poor therapeutic effect, and he has found that all the patients had MGD, most of which have meibomian gland atrophy and deletion; he suggested that there was a significant correlation between MGD and RCES. In 2014, scholars studied 100 RCES patients (117 eyes), which revealing that 60 percent of the RCES eyes were with moderate or severe MGD simultaneously that need to be treated. The most susceptible corneal erosion area is the most susceptible corneal epithelium area that the meibomian gland and dry eye are affected. It was believed that the levels of matrix metalloproteinase (MMP) caused by meibomian gland dysfunction were involved in the occurrence of RCES ${ }^{[8]}$. In our research, it was also found that all patients of RCES had different degrees of meibomian gland dysfunction, the blepharolipin quality was lower than that of the control group, and the blepharolipin score was significantly higher than that of the control group; it was presented that meibomian gland dysfunction may be involved in the pathogenesis of RCES. This is consistent with the previous reports.

MGD was caused by various factors such as infection, inflammation, neurosecretory disorders, congenital abnormalities, and stellwag, while blepharitis would lead to severe MGD. It has been reported within China and overseas that there is a close relationship between the Demodex and anterior blepharitis; the Demodex was positively correlated with the symptom and evaluated scores; control the Demodex can significantly improve the symptoms of blepharitis ${ }^{[9.10]}$. Studies have suggested that the main cause of meibomian gland obstruction is hyperkeratosis of the terminal duct ${ }^{[11,12]}$ and opening. Meanwhile, the Demodex can be the bacteria carrier, bringing the bacteria to the palpebral edge during the process of secreting towards the palpebral edge. Plus, the 
decomposed esterase produced by bacteria may decompose the meibum into free fatty acids and other toxic medium, which will induce subclinical inflammation and release the pro-inflammatory factors, leading to increases the meibum viscosity or promotes epithelial keratinization of the glands, and cause the meibomian gland obstruction ${ }^{[13]}$. Therefore, Demodex also plays a vital role in the pathogenesis of MGD. We also found that almost all the RCES patients have angiotelectasis of the palpebral edge and hyperkeratosis of the meibomian gland opening; multiple forms of secretions can be found attached in the root of most patients' eyelash. All of the above are the characteristics of Demodex-infected blepharitis ${ }^{[14]}$, the detection rate of palpebral edge Demodex in these patients was much higher than that of normal patients. In our study, the detection rate of Demodex in the normal control group was only $38.1 \%$, which was similar in previous literature ${ }^{[15]}$, while the detection rate of Demodex in RCES patients was $85 \%$; this indicated that Demodex infection was very common in RCES patients. Chen $\mathrm{Di}^{[7]}$ found in their study that the positive rate of Demodex in MGD patients was $86.4 \%$. This also implied that a Demodex infection of the palpebral edge is involved in the pathogenesis of MGD and may be directly or indirectly involved in RCES patients' pathogenesis.

The current treatment of RCES mainly includes medication, wear corneal contact lens, mechanical and laser puncture of the anterior corneal stroma, and phototherapeutic keratectomy (PTK), etc. The main goal of the treatment is to relieve symptoms and reduce recurrence. Nevertheless, no treatment can prevent recurrence by now, and data shows that all treatments' recurrence rate was still above 20 percent ${ }^{[16-18]}$.

Treatment for MGD aims to ameliorate the $\mathrm{HBCl}$ of meibomian glands, improve the tear film stability, and relieve the patients' discomfort symptoms. The current treatment includes physiotherapy such as clean palpebral edge, hot compress, meibomian glands massage, etc.; medication like artificial tears eye drops, anti-inflammatory medicine, and antibiotic eye drops; along with diet therapy. IPL refers to a mature technology of dermatology for the treatment of skin telangiectasia, erythema, pigmentation, skin aging, and other diseases, and widely used for its good therapeutic effect. Toyos ${ }^{[19]}$ et al. found that IPL improved patients' ocular surface with facial acne, and they proposed IPL as a potential treatment for MGD. Previous studies have indicated that IPL can be absorbed selectively by melanin and heme in deep skin tissues, causing the destruction and decomposition of pigment groups, the coagulation of blood vessels, and the

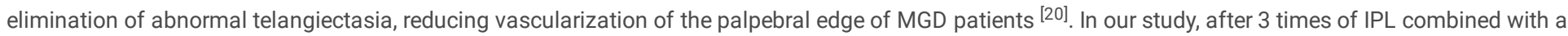
meibomian gland massage treatment, we found that the congestion of the palpebral edge was reduced, the secretion of meibum was significantly improved,

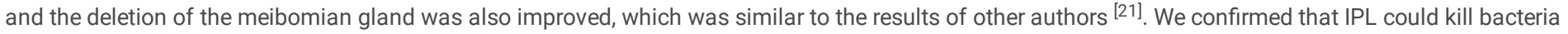
and Demodex ${ }^{[22]}$. Although Demodex still exist after three times of IPL treatment, the number of Demodex has decreased than before. Conversely, it is still unknown whether the palpebral edge's recovery is related to decreasing the Demodex number.

In our study, the ultimate goal of the treatment of MGD was to cure RCES. Surprisingly, we discovered that after 3 times treatments of IPL with meibomian gland massage therapy, the meibomian gland function and the amount of Demodex were significantly decreased, and corneal erosion of the patients has been effectively controlled. Plus, throughout the follow-up period, there was just 1 case that relapsed again. However, the recurrence rate was significantly less than before; the patients have only the corneal epithelium's punctate turbidity, compared to the recurrence before when the patients performed a large corneal epithelial defect, the improvement was remarkable. Our treatment was more effective than drug-only treatment. Our results showed that the treatment of MGD and Demodex is capable of effectively controlling RCES, which may offer a new approach to non-invasive treatment for the clinical treatment of RCES.

It is generally acknowledged that after 3 times of IPL treatment, the therapeutic effect can last 6 months to 1 year. Our study's follow-up period was 1 year; during this period, the disease was effectively controlled. Yet, our next study will be focused on whether there will be relapse as the follow-up period extends. Meanwhile, on the therapeutic mechanism of RCES, whether treatment of IPL is only to improve the meibomian gland function and reduce the amount of Demodex is the direction of our future research.

\section{Declarations}

\section{Ethics approval and consent to participate}

This retrospective study was performed in accordance with the Declaration of Helsinki and was approved by the Ethics Committee of Wuhan Aier Eye Hanyang Hospital (HYEYE2018IRB01). All participants provided written informed consent.

\section{Consent for publication}

Not applicable.

\section{Availability of data and materials}

The datasets generated and analysed during the current study are not publicly available due trade secrets but are available from the corresponding author on reasonable request.

\section{Competing interest}

The authors declares that they have no competing interests.

\section{Funding}

The authors do not have any sources of support, including sponsorship or sources of material not available commercially.

\section{Authors' contributions}

Man Xu: performed the experiment and the data analyses; 
Xiaoguang Niu: performed the experiment and wrote the manuscript

\section{Acknowledgements}

We gratefully acknowledge all of the participants in this study.

\section{References}

1. Xu K, Kam KW, Young AL, et al. Recurrent Corneal Erosion Syndrome. Asia Pac J Ophthalmol (Phila). 2012,1(6):349-54.

2. Hisataka Nanba, Tatsuya Mimura, Yoshinobu Mizuno, et al. Clinical course and risk factors of recurrent corneal erosion: Observational study[J]. Medicine, 2019, 98(16):14964.

3. Geerling G, Lisch W, Finis D. Recurrent Corneal Erosions in Epithelial Corneal Dystrophies. Klin Monbl Augenheilkd. 2018, $235(6): 697-701$.

4. Hope-Ross MW, Chell PB, Kervick GN, et al. Recurrent corneal erosion: clinical features. Eye (Lond). 1994;8 (4):373-7.

5. McMahon FW, Gallagher C, O'Reilly N, et al. Exposure of a corneal epithelial cell line (hTCEpi) to Demodex-associated Bacillus proteins results in an inflammatory response. Invest Ophthalmol Vis Sci. 2014, 55(10):7019-28.

6. Lane SS, Dubiner HB, Epstein RJ, et al. A New System, the LipiFlow, for the Treatment of Meibomian Gland Dysfunction. Cornea 2012,31(4):396ه404.

7. Chen Di, Li Rui, Liu Xiaowei, et al. Prevalence and treatment effects of Demodex species in eyelash follicles in patients with meibomian gland dysfunction. Chin J Ophthalmol, 2017,53(3):193-197.

8. Diez-Feijóo E, Grau AE, Abusleme El, et al. Clinical presentation and causes of recurrent corneal erosion syndrome: review of 100 patients. Cornea. 2014,33(6):571-5.

9. McMahon FW, Gallagher C, O'Reilly N, et al. Exposure of a corneal epithelial cell line (hTCEpi) to Demodex-associated Bacillus proteins results in an inflammatory response. Invest Ophthalmol Vis Sci. 2014, 55(10):7019-28.

10. Sun Xuguang, Zhang Xiaoyu. Discussion on Demodex infection and blepharitis. Chin J Exp Ophthalmol, 2016,34(6):481-483.

11. Liang L, Ding X, Tseng SC. High prevalence of demodex brevis infestation in chalazia. Am J Ophthalmol. 2014,157:342-348.

12. Yu Ting, Hong Jing. Current and emerging treatment options for meibomian gland dysfunction. Chin J Exp Ophthalmol, 2018,36(2):150-155.

13. Kasetsuwan N, Kositphipat K, Busayarat M, et al. Prevalence of ocular demodicosis among patients at Tertiary Care Center, Bangkok, Thailand. Int J Ophthalmol. 2017,10(1):122-127.

14. Zhang Yang, Sun Xuguang. Research on the relationship between clinical features of patients with Demodex blepharitis and diagnosis. Chin J Exp Ophthalmol, 2018,36( 9 ): 688-692.

15. esolowska M, Knysz B, Reich A, et al. Prevalence of demodex spp. in eyelash follicles in different populations[J]. Arch Med Sci, 2014, 10(2):319-324区

16. J Mehlan, J Steinberg, L Traber, et al. Recurrence rate and subjective symptoms after standardized (Hamburg protocol) phototherapeutic keratectomy on recurrent corneal erosions. Graefes Arch Clin Exp Ophthalmol, 2016, 254(10):2005-2009.

17. ReevesSW, KangPC, ZlogarDF, et al. Recurrent Corneal Erosion Syndrome: A Study of 364 Episodes. Ophthalmic Surg Lasers Imaging. 2010,9(3):1-2.

18. Chan $\mathrm{E}$, Jhanji V, Constantinou M, et al. A randomised controlled trial of alcohol delamination and phototherapeutic keratectomy for the treatment of recurrent corneal erosion syndrome. Br J Ophthalmol, 2014, 98(2):166-171.

19. Toyos R, McGill W, Briscoe D. Intense pulsed light treatment for dry eye disease due to meibomian gland dysfunction, a 3-year retrospective study. Photomed Laser Surg, 2015,33 (1):41-46.

20. Piccolo D, Di MD, Crisman G, et al. Unconventional use of intense pulsed light. Biomed Res Int, 2014,2014:618206.

21. Song W J, Yan X M. Research progress of intense pulsed light treatment on meibomian gland dysfunction and relevant dry eye diseases. Zhonghua Yan Ke Za Zhi. 2018,54(2):140-143.

22. Farrell HP, Garvey M, Cormican M, et al. Investigation of critical inter-related factors affecting the efficacy of pulsed light for inactivating clinically relevant bacterial pathogens. J Appl Microbiol, 2010,108(5):1494-1508.

\section{Figures}




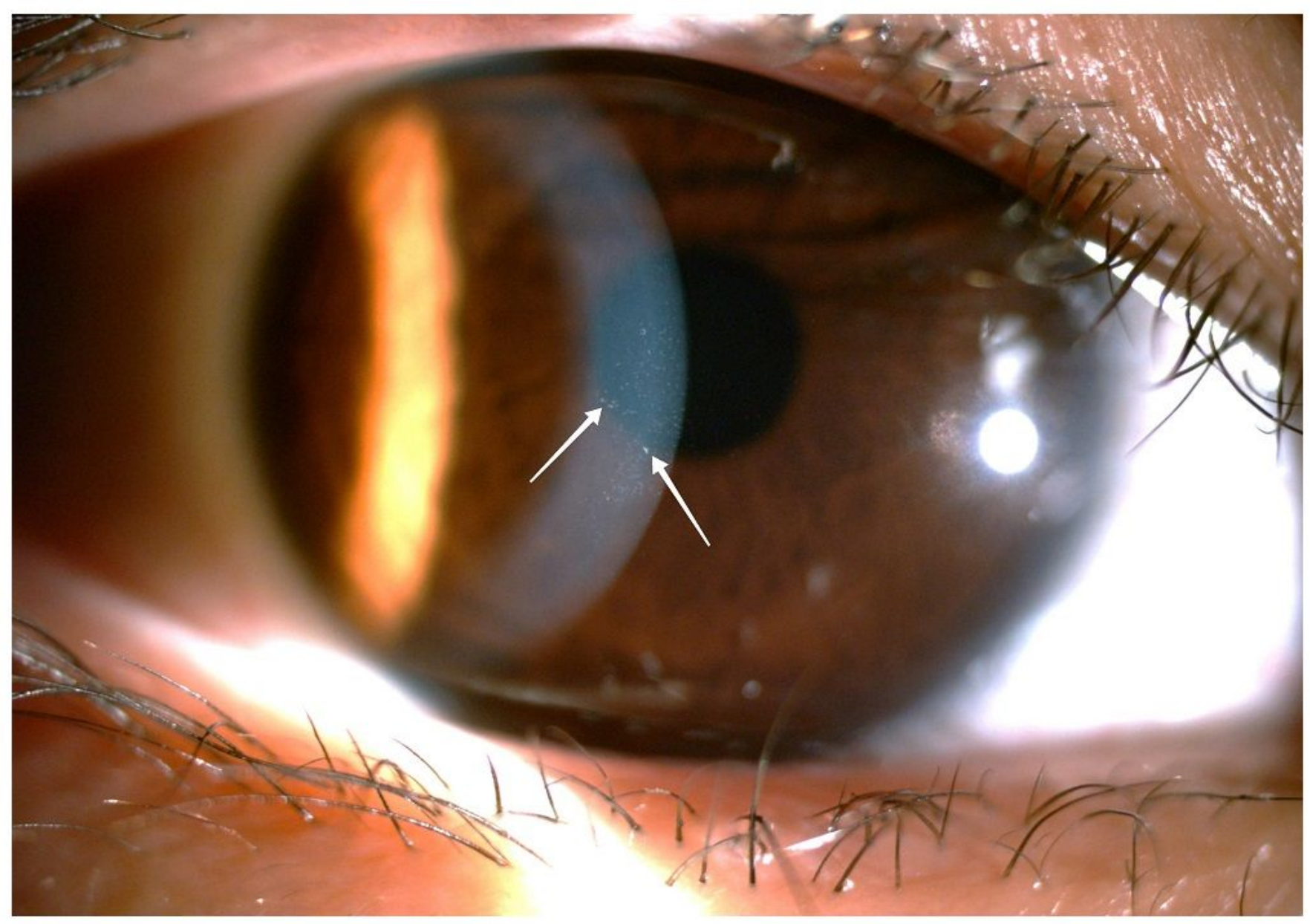

Figure 1

After treatment, the part of the corneal, which the epithelium erosion repeatedly showed punctuate opaque (white arrow), and not detached. 